\title{
Impact of T Helper Cell Subtype on CAR-T Cell Therapy Efficacy
}

\author{
Ibrahim M. Khan, BS BA ${ }^{1}$, \& Andrew S. Nelson, $\mathrm{PhD}^{2}$, Mark H. Kaplan, PhD² \\ ${ }^{1}$ Indiana University School of Medicine. ${ }^{2}$ Wells Center for Pediatric Research, \\ Department of Pediatrics
}

\section{Background and Hypothesis}

Chimeric antigen receptors (CARs) are recombinant receptors with high affinity for the target antigen. Used for tumor therapy, CARs are transduced into patient T cells. CAR-T cells specific for CD19 are used to treat B cell acute lymphoblastic leukemia (B-ALL). Cancerous B cells are destroyed by CAR-T cells in an antigen-specific manner. Currently being used in conjunction with radiation and other cancer therapies to prohibit relapse, Dr. Marco Davila of the Moffitt Cancer Center, has shown that CAR-T therapy induces long term remission and $\mathrm{B}$ cell aplasia.

\section{Experimental Design}

In this experiment the CAR vector obtained from Dr. Davila was transduced into T helper cells cultured under varying conditions (Th0, Th9, and ThGranzyme A). $B$ cell killing and longevity of transduced CAR-Th cells were monitored as part of the criteria for determining the most effective Th subtype for the CAR-T therapy. The target cell killing-mechanism was analyzed at the RNA level using quantitative polymerase chain reaction (qPCR) to analyze gene expression of cytotoxic molecules including granzymes A/B, perforins, Fas-FasL, and TNF- $\alpha$. Th9 cells were expected to be among the most effective of the indicated subtypes due to their longevity and coordination of the immune response.

\section{Results}

T cells in all conditions were effectively transduced for CAR expression, although Th9 cells demonstrated a greater proportion of cultured cells that were transduced with the CAR. QPCR results suggest that there is specification of cytotoxic programs among the culture conditions. In Th9 cells, qPCR results suggest their use of perforin and TNF- $\alpha$. Ongoing studies will compare cytotoxic activity.

\section{Potential Impact}

Further steps after determining the most effective culture conditions include injecting transduced Th cells of the optimized subtype into mice afflicted with BALL to assess cancer killing in vivo as well as the potential harm of the therapy to the patient. 